\title{
Dynamic Link Adaptation and Routing Protocols in Wireless Sensor Networks based on Energy Harvesting of MAC Protocols
}

\author{
Priya Udhawani \\ PG Fellow, \\ Smt. Kashibai Navale College of Engineering \\ Affiliated to University of Pune, Pune.
}

\author{
S.K.Pathan \\ Assistant Professor, \\ Smt. Kashibai Navale College Of Engineering \\ Affiliated to University of Pune, Pune.
}

\begin{abstract}
The design of energy harvesting device is main part of MAC design. Recently the efficient method for energy harvesting (EH) device design for MAC protocols of WSN is studied. Practically this method proven it's efficient in terms of delivery ratio as well as energy consumption. This approach is focused on system-level considerations for networks operating with $\mathrm{EH}$ devices, by addressing the analysis and design of medium access control (MAC) protocols for single-hop wireless sensor networks, where a fusion center (FC) collect the data from sensors in its surrounding. Specifically, we investigate how performance and design of MAC protocols routinely used in WSNs, such as TDMA, framed-ALOHA (FA) and dynamic-FA (DFA). The energy efficiency is improved by using this MAC protocol with energy harvesting. However we can still further improve this method of energy harvesting with MAC protocol by introducing the use of energy efficient routing protocols along with link adaptation technique. Efficient routing algorithm can save a significant amount of energy in a network where routing occurs frequently. In WSN, routing protocols also plays important part for efficient energy utilization. In this paper we are further extending this energy efficient design of $\mathrm{EH}$ device for MAC protocols by adding dynamic link adaptation technique and the energy efficient routing protocol to it.
\end{abstract}

\section{Keywords}

Medium Access Protocol, Energy Consumption, Energy Harvesting, Cluster, Network lifetime, Routing Protocols, TDMA, ALOHA.

\section{INTRODUCTION}

Energy is limited resource for every sensor node in WSNs which decides the lifetime of overall sensor network. Therefore WSN must need to have energy saving methods in place to save the consumption power during the different activities of sensor nodes. Management of the radio transceiver unit of a wireless device has gained significant importance with the emerging of wireless sensor networks since the radio unit is the major consumer of the sensor's energy. It has been shown that the energy consumed in transmitting one bit is several thousand times more than the energy consumed in executing one instruction. Since the radio transceiver is the major power consumer unit and the MAC protocol directly controls its operation, several MAC layer protocols have been proposed to reduce the energy consumption of the sensor's radio unit. For some examples refer to reference, which surveys a large set of MAC protocols designed specifically for WSNs [1]
Energy consumption has been considered as the single and important design key in sensor networks, hence, the most recent work on medium access control (MAC) protocol for sensor networks focused on energy efficiency, where MAC protocols play a crucial role in controlling the usage of the radio unit. The radio transceiver unit is the major power consumer unit in the sensor node. For most MAC protocols designed for WSNs, it is assumed that the sensor nodes are stationary, which causes performance degradation when these protocols are applied in mobile environments.

In WSNs for better energy management, the concept of energy harvesting technologies used for MAC protocols. The recent method introduced in [1], in which the focus was on system level design of WSN those are operating with using EH devices for the efficient design of MAC protocols in WSNs. In this architecture, fusion center (FC) is collecting the data from the sensor nodes in its proximity. The performance of this approach is investigated with different MAC protocols those are influenced by discontinuous availability of energy in EH devices [1]. However this method was not tested by using the routing protocols considerations which are playing the vital role for energy utilization in WSNs. Therefore in this paper we are presenting the extension to this work by using the recent energy efficient routing protocol i.e. LEACH and HEED protocols along with dynamic link adaptation method with above stated energy efficient architecture design using EH devices to delivers the best energy efficiency for WSNs.

In next section 2 we are presenting the literature survey over the various methods for energy efficiency in WSNs. In section 3 , the proposed approach and its system block diagram is depicted. In section 4 we are presenting the implementation details and results achieved. In section 5, the results are discussed. Finally conclusion and future work is predicted in section 6 .

\section{LITERATURE SURVEY}

In this section we are presenting the different methods those are presented for efficient MAC protocols and survey over energy harvesting.

\section{1: Energy Harvesting Review}

- In [1], author presented the design of medium access control protocol by using the $\mathrm{EH}$ device designing in order to achieve the efficient energy utilization. However this method is required to be focused by using routing protocols those are energy efficient.

- In [2], the authors presented the various aspects of energy harvesting systems. Also presented basic 
concepts of harvesting systems - architectures, types of harvestable energy sources, and storage technologies. Also described details of existing energy harvesting sensor nodes and applications, most notably the ones dependent on solar energy.

- In [3], presented the WSN architecture for habitat monitoring and its implementation. With the advancement in the internet technology provides a connectivity of application and information sharing in the Habitat monitoring. The protocol design for energy harvesting is a challenging task. In this paper, authors discussed the various methods and challenges of recent development in the harvesting energy and the research scope.

\section{2: Energy Efficient MAC and Routing Protocols}

- B-MAC Protocol: Berkeley Media Access Control (BMAC) which is widely used in WSNs. B-MAC is also similar to CSMA protocol having a feature of Low Power Consumption. With filter mechanism, it increases reliability and channel assessment. BMAC employs an adaptive preamble sampling scheme which minimizes idle listening and reduce duty cycle. B-MAC duty cycles the radio through periodic channel sampling that are called Low Power Listening (LPL). The clear channel assessment (CCA) technique is used by BMAC to decide if a packet is arriving when node wakes up. If no packet arrived timeout puts node back to sleep. CCA and packet backoffs are used by BMAC for channel arbitration, link layer acknowledgments for reliability. There are no synchronization, RTS, CTS in BMAC.

- S-MAC Protocol: This protocol is a contention based protocol. This protocol essentially trades energy for throughput and latency by utilizing the sleep mode of the radio. The sensor node periodically goes to the fixed sleep cycle and the time frame is divided into two parts: one for a listening session and the other for a sleeping session. In SMAC, the sensor node are capable to communicate with additional nodes and sends some control packets such as SYNC, RTS (Request to Send), CTS (Clear to Send) and ACK(Acknowledgement) [4] .

- T-MAC Protocol (T-MAC) is proposed to enhance the poor results of S-MAC protocol under variable traffic load. In T-MAC, when no activation event has occurred listen period ends for a time threshold. T-MAC is proposed to enhance the poor results of S-MAC protocol under variable traffic load [5].

- PAMAS Protocol: The Power Aware Multi Access with Signaling protocol is based on the MACA protocol but includes separate signaling channel to avoid the collision and overhearing problem. All nodes utilize the signaling channel to exchange RTSCTS frame and therefore gain access to the media. All nodes know who has gained the media and for how long, information that nodes use to turn themselves off. The other channel is use exclusively to transmit data frame which is collision-free. The main disadvantage of PAMAS is that it needs an additional radio for signaling channel, which adds to the cost of sensor network devices[6].
- LEACH protocol: Heinzelman et al. proposed an alternative clustering-based algorithm, called LEACH (Low-Energy Adaptive Clustering Hierarchy). It assumes that there exists a unique base station outside the sensor network and all the sensor nodes can communicate with this base station directly. LEACH includes the idea of clustering but without including any powerful node as the cluster $h$ ead. Second, in order to save additional energy in the nodes LEACH utilizes TDMA schedule based MAC mechanisms for intracluster communications. In order to save energy, LEACH only chooses a fraction $p$ of all sensor nodes to serve as cluster heads, where $\mathrm{p}$ isa design parameter that must be determined before deployment. The rest sensor nodes join the proper clusters according to the signal strength from cluster heads. In order to share the energy load, its operation is divided into rounds which can guarantee the cluster head rotate in each round. In each round, after cluster formation phase, the cluster heads aggregate the data received from their cluster members and send the aggregated data to the base station by single hop communication, so it can sharply reduce the data needed to be transmitted to the base station [7][12].

- HEED Protocol: The authors proposed a hybrid, energyefficient, distributed clustering algorithm (HEED) which periodically selects cluster head according to a hybrid of the node residual energy and a secondary parameter such as node proximity to its neighbors or node degree. The aim of the algorithm is to prolong the lifetime of a WSN. Heed terminates in $\mathrm{O}(1$ iterations and incurs low message overhead. It achieves fairly uniform cluster head distribution across the network [8].

\section{PROPOSED APPROACH FRAMEWORK AND DESIGN}

\section{1: Problem Definition}

Recently we have studied one efficient method for energy harvesting $(\mathrm{EH})$ device design for MAC protocols of WSN [1]. Practically this method proven it's efficient in terms of delivery ratio as well as energy consumption. Basically in this [1] author focused on system-level considerations for networks operating with $\mathrm{EH}$ devices, by addressing the analysis and design of medium access control (MAC) protocols for single-hop WSNs, where a fusion center (FC) collects data from sensors in its surrounding. Specifically, we investigate how performance and design of MAC protocols routinely used in WSNs, such as TDMA, framed-ALOHA (FA) and dynamic-FA (DFA). With all this protocol this proposed method improves the energy efficiency. However we can still further improve this method of energy harvesting with MAC protocol by introducing the use of routing protocols. Routing is an essential feature in any multihop sensor network. In WSN, routing protocols also plays an important part for efficient energy utilization. Dynamic link adaptation method with routing protocols are not focused by author and may be the further research area to extend this method. 


\section{2: Proposed Design}

In Wireless Sensor Networks (WSN), energy is critical resource and constraint of such networks. There are number of researches already done on minimizing the energy consumption of WSN in order to extend the lifetime of entire network. Recently different methods introduced which were working over MAC layer protocols of WSN. These approaches are based on use of energy harvesting (EH) device[10]. In this project first we are investigating one such efficient method in which EH devices are designed for WSN. This method aimed to address the analysis and design of WSNs with EH devices by focusing on conventional MAC protocols, namely TDMA, framed-ALOHA (FA) and dynamic-FA (DFA), and by accounting for the performance trade-offs and design issues arising due to $\mathrm{EH}$. In addition to this method, we are further extending this approach by adding the energy efficient routing protocol called HEED rather than using LEACH and using the dynamic link adaptation technique. Routing protocol is also playing major role for the consumption of energy in WSN. Hence in this project we are basically extending the current approach of MAC protocols with EH by using the HEED with link adaptation technique. The performance parameters which we are taking here under investigation are energy consumption, packet delivery ratio, throughput to claim proposed approach efficiency [11][12].

Figure 1 below showing the proposed EH design for WSNs. We consider a WSN with a Fusion Center surrounded by wireless sensor nodes.

1. Management of Inconsistence: It is vital to have a mechanism to manage and control the quality of data collected by wireless sensor network. Since there would be data loss resulting from unreliable wireless communication.

2. Management of Dynamic Usermodel: The location where the sensor data is stored or is the dynamic data storage in which the location of the repository is chosen based on information collected over the network. It chooses to store sensed data close to where it is most frequently needed or close to the query entry point when the query rate is high. It is needed to reduce the power consumption of the network.

3. Open Content Platform: To monitor and control the functionalities. The Platform should be open and support the largest possible number of underlying sensor network technologies.

4. Pre-processing Data Receipt: Raw sensor data will be preprocessed at each node to extract a small set of parameters to be forwarded to fusion center.

5. Data Processing Application: It requires to extract the information that is meaningful. Data processing techniques perform efficiently for their intended WSN application.
6. Data Compress: To encode information with fewer bits than the original representation. It improves the performance and low memory is required.

Following Figure 2 is showing the overall flow of proposed paper approach and architecture:

TDMA: With the TDMA protocol, each user is preassigned an exclusive slot that it can use in every IR, irrespective of whether it has a packet to deliver or enough energy to transmit.

DFA \& FA. Hereafter, we describe the DFA protocol only, since FA follows as a special case of DFA with no retransmissions capabilities as discussed below.

LEACH: Low Energy Adaptive Clustering Hierarchy (LEACH) is a TDMA-based MAC protocol which is integrated with clustering and a simple routing protocol in wireless sensor networks (WSNs). The goal of LEACH is to lower the energy consumption required to create and maintain clusters in order to improve the life time of a wireless sensor network. LEACH is a hierarchical protocol in which most nodes transmit to cluster heads, and the cluster heads aggregate and compress the data and forward it to the base station (sink). Each node uses a stochastic algorithm at each round to determine whether it will become a cluster head in this round. LEACH assumes that each node has a radio powerful enough to directly reach the base station or the nearest cluster head, but that using this radio at full power all the time would waste energy.

HEED: The HEED protocol is an energy-efficient clustering protocol designed for WSNs. The aim of the algorithm is to prolong the lifetime of a WSN. In HEED cluster-head selection is based on two different parameters. The primary parameter is the residual energy of each node, while the second parameter measures the intracluster communication cost, the number of neighbours. The idea is to use the primary parameter to perform a probabilistic choice of an initial set of cluster heads, and the second parameter to break ties between them. This is an iterative algorithm in which nodes change their probability of becoming cluster-head $\mathrm{CHprob}$ at each iteration.

LINK ADAPTATION: Link adaptation techniques answer the questions of What to change? And When to change? in order to improve the present layer performance. Once these decisions are made, other layers are expected to function perfectly with the new communication channel conditions. The research demonstrated in this dissertation contributes to our understanding of link adaptation techniques and broadens the scope of simple, one step physical parameter adjustments[13]. 


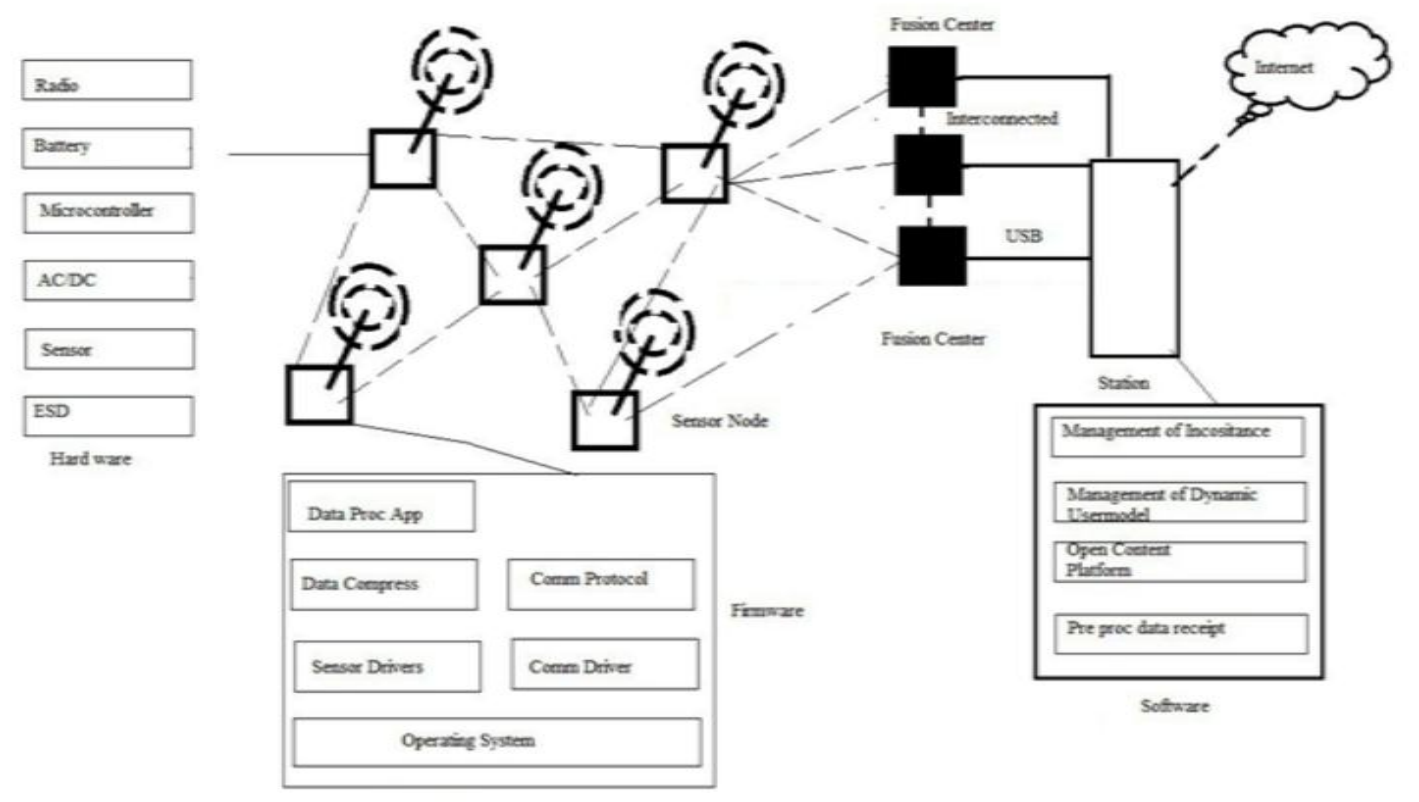

Fig 1: Architectural Design of Proposed EH in WSNs.

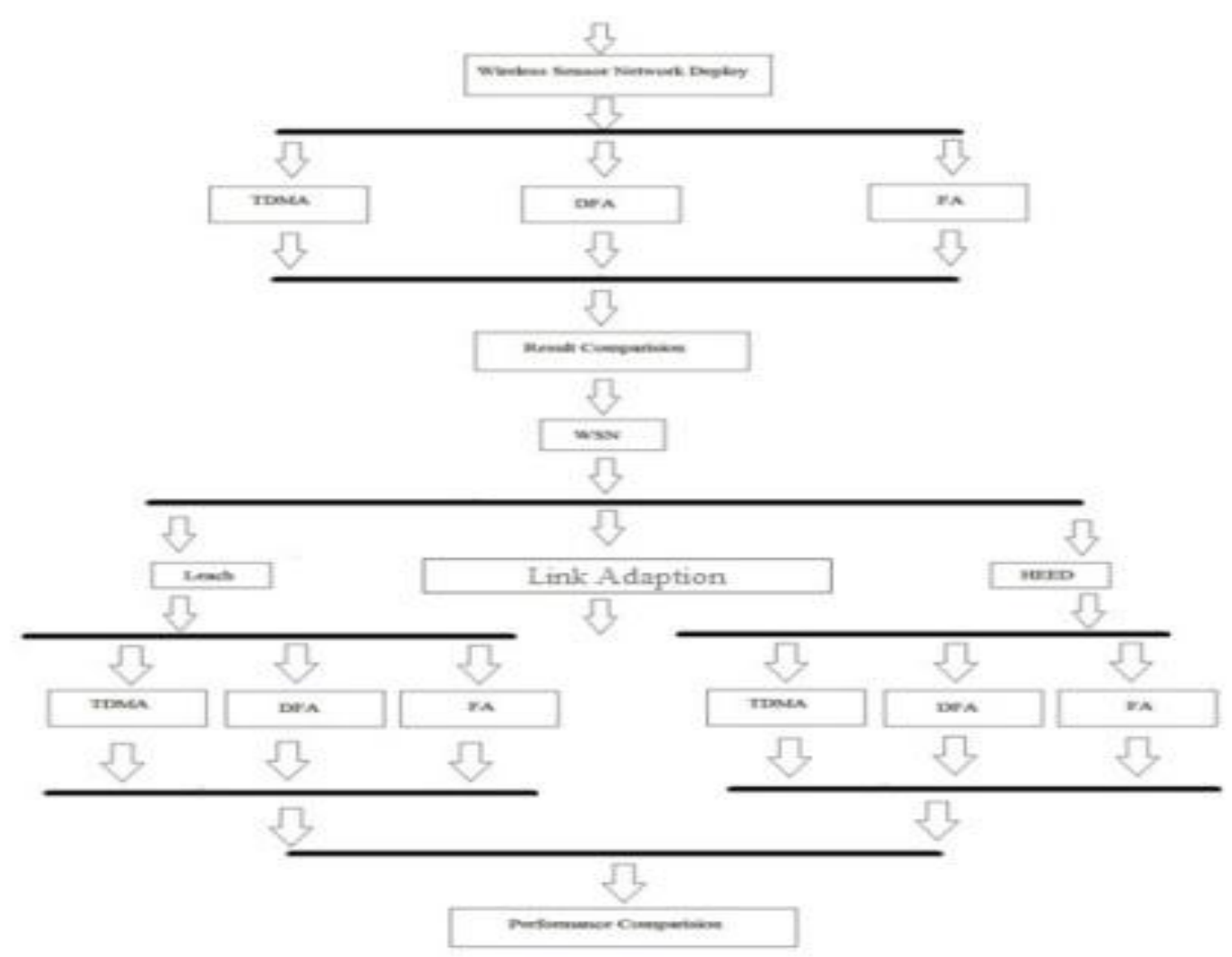

Fig 2: Block Diagram of Proposed System. 


\section{IMPLEMENTATION}

Once the clusters are created and the TDMA schedule is fixed, data transmission can begin. The non-cluster head nodes send data to cluster head node during their allocated transmission time. When all the data have been received, the cluster head node performs signal processing to compress the data into a single signal. Then, this signal is sent to the BS. The amount of information is reduced due to the data aggregation done at the cluster head node.

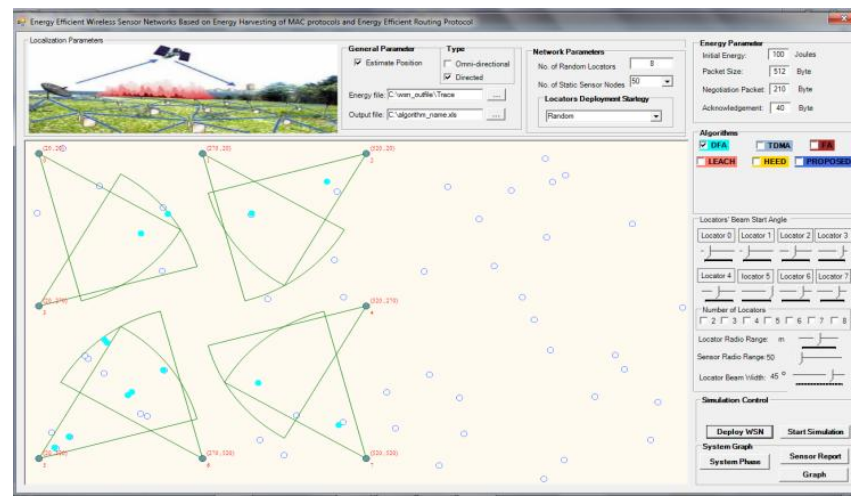

Fig 3: Sensor node with DFA Protocol

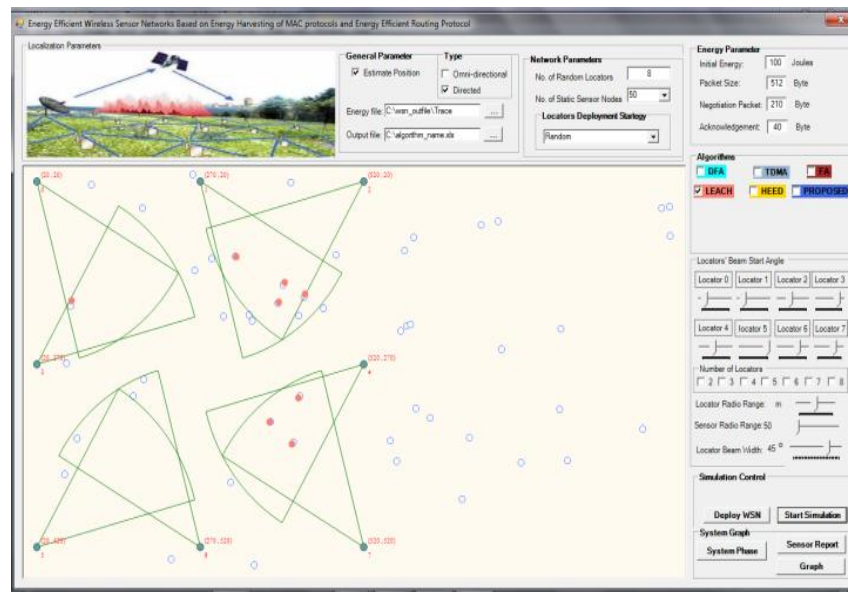

Fig 4: Sensor node with LEACH Protocol

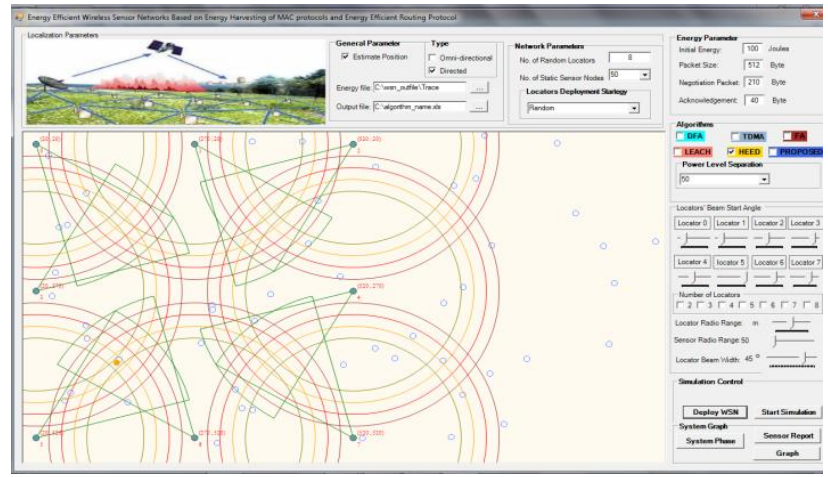

Fig 5: Sensor node with HEED Protocol

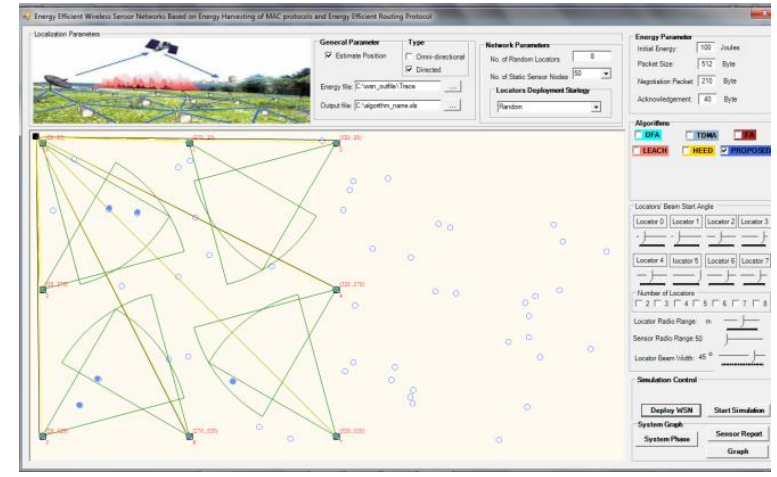

Fig 6: Output Screen of proposed algorithm with dynamic link adaptation

Figure 3 shows the output screen of sensor node with DFA Protocol. Figure 4 shows the output screen of sensor node with LEACH Protocol, Figure 5 shows the output screen of sensor node with HEED Protocol, Figure 6 shows output screen of dynamic link adaptation method. Figure 7 shows the detail phase of the system.

\section{RESULTS AND DISCUSSIONS}

In this section, we present extensive numerical results to get insight into the MAC and Routing protocols design with the dynamic link adaptation method. Figure 8 shows that the proposed algorithm is more energy efficient, as the amount of energy required is reduced.Figure 9 shows the graph of endto-end delay, which refers to time taken for a packet to be transmitted across a network from source to destination. Figure 10 shows graph of the residual energy. Figure 11 shows the packet delivery ratio graph,Packet delivery ratio is defined as the ratio of data packetsreceived by the destinations to those generated by the sources. 


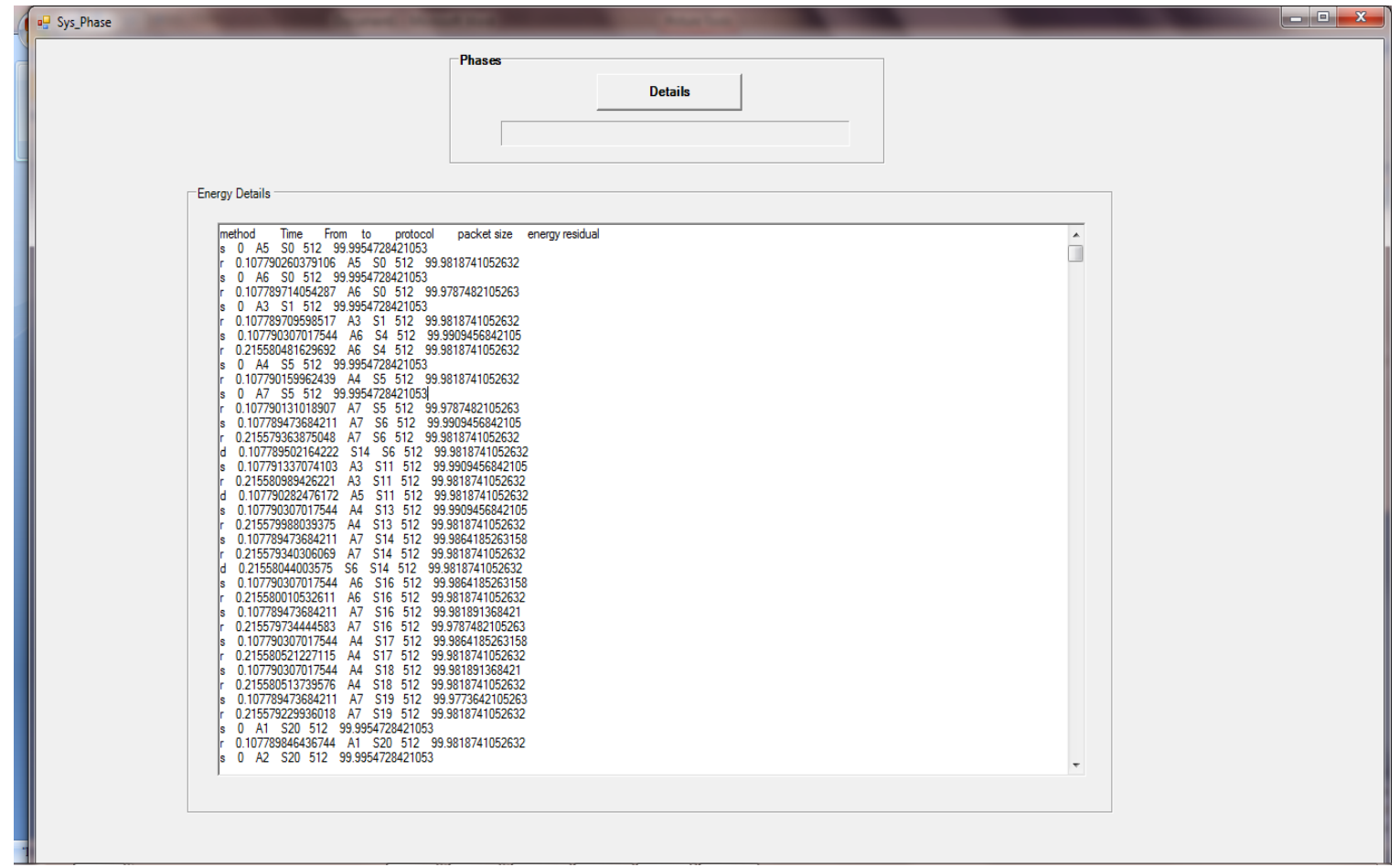

Fig 7: Detail Phase of the system showing source, destination, Protocol Name and residual energy of each node

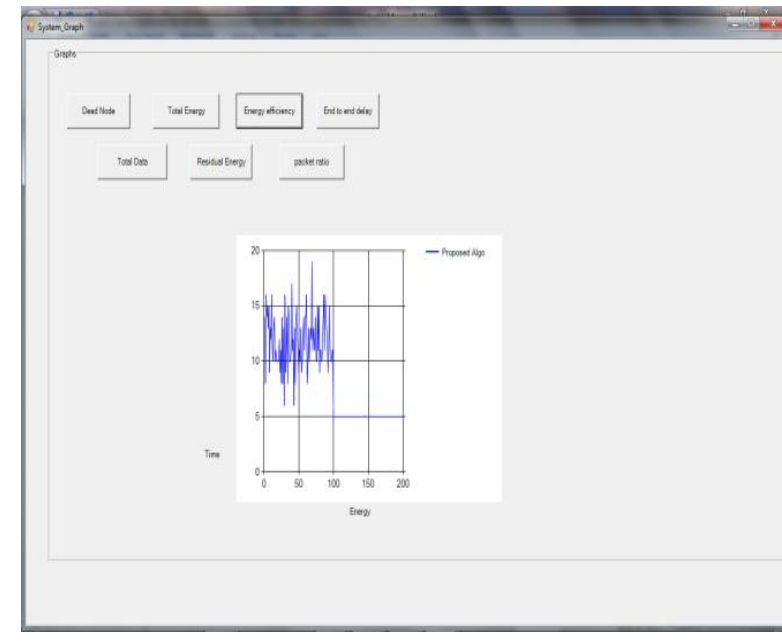

Fig 8: Energy Efficiency graph between time and energy

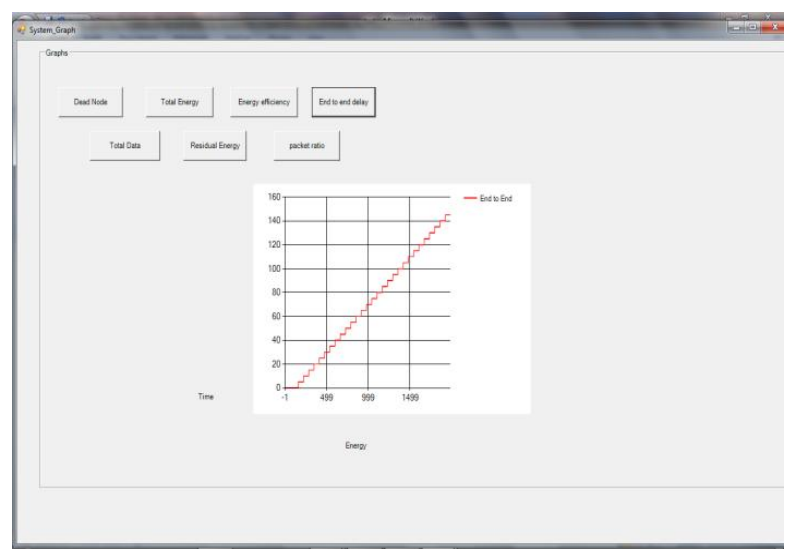

Fig 9: Graph showing the Result of End-to-End Delay

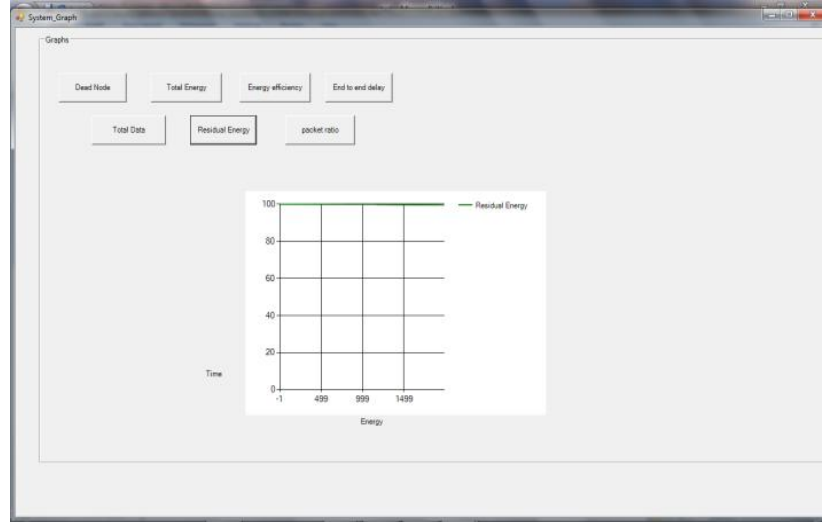

Fig 10: Graph showing Residual Energy

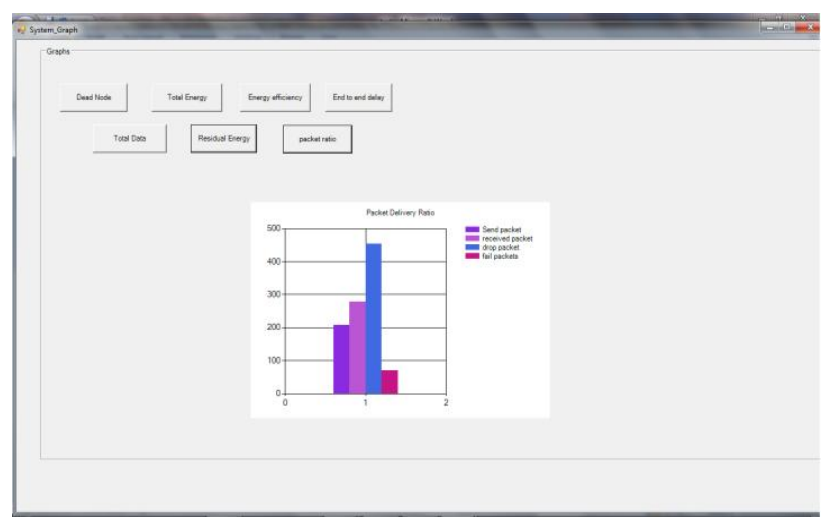

Fig 11: Graph showing Packet Delivery Ratio 


\section{CONCLUSION AND FUTURE WORK}

The management of energy in wireless sensor network is very vital tasks in order to extend the lifetime of sensor networks. There are many approaches and designs already presented for energy efficiency. In this paper we have presented the system level approach for energy management and efficiency using the terminology of energy harvesting device. This devices are designed to use with MAC protocols in order to deliver the best energy efficiency while communication. In addition to this EH and MAC, we have proposed to use energy efficient routing algorithm along with dynamic link adaptation method as well so that overall network performance is increasing. The practical results presented here are the' state of our experimental studies. For the future work we suggest to check these possibilities of energy efficiency using proposed framework under the real time environment.

\section{REFERENCES}

[1] Fabio Iannello, Student Member, IEEE, Osvaldo Simeone, Member, IEEE, and Umberto Spagnolini, Senior Member, IEEE, "Medium Access Control Protocols for Wireless Sensor Networks with Energy Harvesting", IEEE TRANSACTIONS ON COMMUNICATIONS, VOL. 60, NO. 5, MAY 2012.

[2] Sujesha Sudevalayam Purushottam Kulkarni, "Energy Harvesting Sensor Nodes: Survey and Implications", December 19, 2008.

[3] Dr. S. Swapna Kumar*, Dr K.R Kashwan, "Research Study of Energy Harvesting in Wireless Sensor Networks", INTERNATIONAL JOURNAL of RENEWABLE ENERGY RESEARCH et al, Vol.3, No. 3

[4] T. Chiras, M. Paterakis, and P. Koutsakis, "Improved medium access control for wireless sensor networks - A study on the SMAC protocol", In 14

[5] Tijs van Dam, Koen Langendoen," An adaptive energyefficient MAC protocol for wireless sensor networks ",
SenSys '03 Proceedings of the 1 st international conference on Embedded networked sensor systems in ACM conference, pp. 171-180, 2003

[6] S. Singh and C. Raghavendra. PAMAS: Power aware multi-access protocol with signaling for adhoc networks, ACM SIGCOMM Computer Communication Review, 28(3):5-26, July 1998.

[7] W. Ye, J. Heidemann, and D. Estrin, 2002. An energyefficient MAC protocol for wireless sensor networks. In Proceedings of the IEEE Infocom, pp: 1567-1576.

[8] Al-Karaki, J.N.; Kamal, A.E. Routing techniques in wireless sensor networks: A survey, IEEE Wireless Communication, 2004, 11, 6-28

[9] Omeni, O. and Wong, A. and Burdett, A.J. and Toumazou, C, 2008. Energy efficient medium access protocol for wireless medical body area sensor networks IEEE.

[10] J. A. Paradiso and T. Starner, "Energy scavenging for mobile and wireless electronics," IEEE Perv. Comput. Mag., vol. 4, no. 1, pp. 18-27, Jan.-Mar. 2005.

[11] A. Kansal, J. Hsu, S. Zahedi, and M. B. Srivastava, "Power management in energy harvesting sensor networks," ACM Trans. Embedded Computer. System., vol. 6, no. 4, art. 32, Sep. 2007.

[12] I. F. Akyildiz, S. Weilian, Y. Sankarasubramaniam, and E. Cayirci, "A survey on sensor networks," IEEE Communication. Mag., vol. 40, no. 8, pp. 102-114, Aug. 2002.

[13] Yanhua Li, Student Member, IEEE, Abedelaziz Mohaisen, Member, IEEE, and Zhi-Li Zhang, Fellow, IEEE, "Trading Optimality for Scalabilty in LargeScale Opportunistic Routing “, IEEE TRANSACTIONS ON VEHICULAR TECHNOLOGY VOL62, NO. 5, JUNE 2013. 